\title{
Translationally invariant clusters in coordinate space: an Euler-Lagrange approach
}

\author{
R F Bishopł, E Buendiał, M F Flynn $\|$ and R Guardiola \\ $\dagger$ Department of Mathematics, UMIST, PO Box 88, Manchester M60 1QD, UK \\ $\ddagger$ Departamento de Física Moderna, Universidad de Granada, Facultad de Ciencias, \\ 18071 Granada, Spain \\ § Departamento de Física Atómica y Nuclear, Universidad de Valencia Estudi General, \\ Avda. Dr Moliner 50, 46100-Burjasot, Valencia, Spain
}

Received 10 October 1991, in final form 30 March 1992

\begin{abstract}
We have previously formulated a general translationally invariant version of the coupled cluster theory. We investigate here the linearized approximation referred to as the second-order translationally invariant cluster method. In particular, we algebraically formulate and numerically solve this method at the optimal Euler-Lagrange level for finite bosonic systems. The method proves to be highly superior to the standard configurationinteraction calculations of the shell-model type, and confirms the validity of the Gaussian geminal expansion of the two-body cluster $C_{2}$.
\end{abstract}

\section{Introduction}

In the last two years we have made a considerable effort [1-3] to understand the structure and practical viability of coupled cluster or $\exp (S)$ theory [4-6] for finite systems, particularly for very light systems. Coupled cluster theory was reformulated so as to remove, exactly and unambiguously, the spurious centre-of-mass motion which is a perennial problem with practical shell-model calculations. We also paid particular attention to the relation of our formulation [7] to large-scale shell-model calculations [8-10].

The standard formulation of coupled cluster theory starts by considering a space of single-particle orbitals divided into two subspaces. One of them is fully occupied by the $A$ constituents of the many-body system in some non-interacting approximation or other reference state, whereas the complementary orthogonal space is completely empty. The ansatz for the many-body wavefunction is

$$
|\Psi\rangle=\exp (S)|\Phi\rangle \quad S=\sum_{n=1}^{A} S_{n}
$$

where $|\Phi\rangle$ is the reference state and the cluster correlation operator $S$ is the sum of one-body, two-body ... operators whose action is to promote one-particle, twoparticles ... from the reference subspace to the empty subspace. The power of

II Current address: Department of Physics and Liquid Crystal Institute, Kent State University, Kent $\mathrm{OH} 44242$, USA. 
this ansatz is that for infinite (extended) systems the wavefunction incorporates from the very beginning the properties of linkedness (or conectedness) and irreducibility required by the Goldstone theorem. These properties do not apply in the case of a finite system. Nevertheless, when the system has a large number of constituents, its properties must approach those of the infinite system. Thus, it is reasonable to start with a description such as that in (1), which has a smooth transition to the limit of infinite systems.

On the other hand, there are two other properties which are required for the proper description of a finite system, namely translational invariance and rotational invariance. The incorporation of the latter is well known in shell-model theories. For example, one may start from a closed-shell wavefunction and afterwards apply $n$-particle- $n$-hole $(n \mathrm{p}-n \mathrm{~h})$ operators, with $n=1,2 \ldots$, which are scalars under rotations. The equivalent in an extended system is that the sum of single-particle momenta produced by a given $n \mathrm{p}-n \mathrm{~h}$ excitation is zero. In the case of extended systems this mechanism also maintains the translational invariance, but this is not the case in finite systems, for which an improper treatment of the translational invariance leads to problems with the centre-of-mass motion.

In our previous work [1-3] we have found a way of dealing with the centre-ofmass problem for finite systems within the coupled cluster theory framework. The most important findings of our work were as follows.

(i) The single-particle space must be the space of eigensolutions of an isotropic harmonic oscillator.

(ii) The clusters appearing in equation (1) must be redefined. In the first place, there cannot appear an $S_{1}$ cluster, which will violate the translational invariance. Moreover, any generic cluster operator $S_{n}$ must produce, in addition to $n \mathrm{p}-n \mathbf{h}$ excitations, an admixture of $m p-m h$ excitations, with $m=1,2, \ldots, n-1$, in a predetermined ratio, in order to ensure the translational invariance.

(iii) The coupled cluster ansatz should be modified by adding a normal-ordering prescription to the structure of the wavefunction, ie.

$$
|\Psi\rangle=: \exp (S):|\Phi\rangle
$$

These results, particularly the first two, have the effect of simplifying in a very significant way the structure of the wavefunction and the solution of the related Schrödinger equation. For example, with respect to the (bosonic) $A$-body reference state

$$
|\Phi\rangle=(A !)^{-1 / 2}\left(a_{000}^{\dagger}\right)^{A}|0\rangle
$$

where $|0\rangle$ is the vacuum state, and the operators $a_{n l m}^{\dagger}$ create single-particle (harmonic oscillator) states with quantum numbers $(\mathrm{nlm})$, the new $S_{2}$ cluster correlation operator has the general structure

$$
S_{2}=\sum_{n=1}^{\infty} S_{2}^{(n)} \theta_{n}
$$

with

$$
\theta_{n}=\sum_{n_{1} n_{2} l m}\left\langle n_{1} l n_{2} l 0 \mid n 0000\right\rangle\langle l m l-m \mid l l 00\rangle a_{n_{1} l m}^{\dagger} a_{n_{2} l-m}^{\dagger} a_{000}^{2}
$$


where the Brody-Moshinsky bracket comes from the requirement of translational invariance, and the Clebsch-Gordan coupling is related to the rotational invariance. The simplification lies in the fact that the amplitudes to be determined, $S_{2}^{(n)}$, have only one index, instead of the three indices $\left(n_{1}, l, n_{2}\right)$ which will appear in the standard formulation of coupled cluster theory with the single requirement of rotational invariance.

We should mention, however, that the third point mentioned above, i.e. the presence of the normal-ordering operator, poses a new problem for solving the Schrödinger equation in this coupled-cluster formulation. The usual way [4] is to project the transformed equation

$$
\exp (-S) H \exp (S)|\Phi\rangle=E|\Phi\rangle
$$

onto the various $m p-m \mathrm{~h}$ subspaces, with $m=0,1,2, \ldots$ In this way one obtains a hierarchy of finite-order non-linear equations relating the unknown amplitudes and the energy. The relevant equations are easily obtained by using the standard nested commutator expansion

$$
\exp (-S) H \exp (S)=H+[H, S]+[[H, S], S] / 2 !+\cdots
$$

which is, however, no longer valid in the presence of the normal-ordering requirement. We have not yet fully solved this additional complication.

In spite of this pending question, the coupled-cluster ansatz may be used to tailor approximate wavefunctions which are expected to be very accurate in finite systems. For example, a very simple form is

$$
|\Psi\rangle=\left(1+C_{2}\right)|\Phi\rangle
$$

which contains only $2 \mathrm{p}-2 \mathrm{~h}$ excitations (mixed, as said above, with $1 \mathrm{p}-1 \mathrm{~h}$ excitations). This simple form permits a variational estimation of the ground state energy and is a counterpart of the traditional configuration-interaction shell-model calculations.

The wavefunction given in (8) describes the system in a form which may be called linear coupled-cluster theory or, more precisely, the second-order translationally invariant cluster ( $\mathrm{TIC}\left[C_{2}\right]$ ) method. Actually, this wavefunction selects the configurations relevant to the system in a very different way from the criterion normally used in configuration-interaction (CI) calculations $[10,11]$, while still maintaining the translational invariance of the wavefunction. Thus, instead of choosing complete $n \hbar \omega$ subspaces, as normally used in shell-model calculations, the $\operatorname{Tic}\left[C_{2}\right]$ form selects only one state from each subspace of $n$ excitation quanta, where $n$ is even by parity symmetry requirements.

Our previous calculations using the TIC $\left[C_{2}\right]$ ansatz [1] were carried out directly in the harmonic oscillator basis, and with use of the standard shell-model machinery. Two main results may be extracted from this work. Firstly, it was clear that the $\mathrm{TIC}\left[C_{2}\right]$ way of selecting the interacting configurations is indeed a very clever way of doing it. Indeed, with bases of relatively low dimensionality (e.g. up to 30 states, equivalent to the inclusion of configurations up to $60 \hbar \omega$ of excitation energy), there result values for the ground state (GS) energy [7] significantly lower than analogous $\mathrm{CI}$ calculations involving thousands of states $[8,10]$. Moreover, this form is appropriate even for two-body interactions with soft but infinite cores, like yukawians. The second 
result is less immediately encouraging. Thus, it turned out that the expansion of the GS energy in this basis was very slowly convergent. In particular, when considering interactions showing strong repulsion at short distances, like the S3 interaction of Afnan and Tang [12] or the MTV interaction of Malfliet and Tjon [13], we were not able to obtain fully converged results. On the other hand, we should mention that in so far as the method is truly variational, the energy lowers continuously when increasing the number of basis states.

The obvious conclusion is that the harmonic oscillator basis is not appropriate to describe the interacting configurations, so that one should abandon it. We recall only that the harmonic oscillator basis was required (at the algebraic level) because it is the only basis capable of producing translationally invariant states within the shell-model description. However, equation (8) may be simply written in a way independent of the single-particle basis by moving to the coordinate representation [1], with the result

$$
\Psi\left(\boldsymbol{r}_{1}, \ldots, \boldsymbol{r}_{A}\right)=\sum_{i<j=1}^{A} f\left(r_{i j}\right) \Phi\left(\boldsymbol{r}_{1}, \ldots, \boldsymbol{r}_{A}\right)
$$

where $\Phi\left(r_{1}, \ldots, r_{A}\right)$ is the harmonic-oscillator uncorrelated wavefunction appropriate for the system of $A$ particles. The quantity to be determined variationally is the function $f(r)$, instead of the coefficients of its expansion in a harmonic-oscillator basis.

A method which we have found to be very efficient in dealing with this problem $[14,15]$ is to use a Gaussian geminal basis [16-18] to expand $f(r)$. Nevertheless, it is quite obvious that the optimal pair correlation function $f(r)$ should be determined through the solution of the integro-differential equation resulting from the Euler-Lagrange variational condition applied to the energy functional. This is the objective of this paper. Section 2 is devoted to a precise statement of the problem and its formulation via an integro-differential equation. In section 3 the kernels corresponding to the potential energy operator and the identity operator, required for the normalization, are obtained. The kinetic energy kernel is converted in section 4 into an algebraically equivalent form of the normalization kernel and its derivatives. Section 5 describes briefly the numerical techniques used to solve the resulting integro-differential equation. Finally, section 6 deals with the results and conclusions of our work. Some technical questions are clarified in the appendix. This work has been carried out for finite boson systems. Some further non-trivial steps are required to deal with fermion systems.

\section{The Euter-Lagrange formulation of the second-order $\mathrm{nc}\left[C_{2}\right]$ approximation}

The underlying motivation for the determination of the Euler-Lagrange equations in the linear coupled-cluster second-order approximation lies in the simple structure of the wavefunction in the coordinate space representation of equation (9).

The reference state $\Phi\left(r_{1}, \ldots, r_{A}\right)$ is the harmonic-oscillator uncorrelated wavefunction appropriate for the system of $A$ particles, and is simply the product of single-particle $0 s$ harmonic oscillator states in the case of bosonic systems, or a Slater determinant for fermion systems. The role of this function is simply to localize the system, as well as to impose the correct overall symmetry or antisymmetry properties. This function is characterized by a single parameter corresponding to the harmonic 
oscillator spring constant, and which will be considered as a variational parameter. It is worth mentioning that the harmonic oscillator structure of the uncorrelated wavefunction was required both to obtain the coordinate-space representation of the full wavefunction from its Fock space form, as well as to ensure the translational invariance of the physical wavefunction, defined implicitly by equation (9) by removing (uniquely) the centre-of-mass motion $[1,7]$.

The other quantity needed to fully characterize the physical wavefunction is the $C_{2}$ cluster or its equivalent pair correlation function $f\left(r_{i j}\right)$, which will be considered as an arbitrary function with the only restriction of having a square integrable wavefunction for the $A$-particle system. In our previous work the correlation function was parametrized as a linear combination of Gaussians,

$$
f(r)=\sum_{k} A_{k} \exp \left(-b_{k} r^{2}\right)
$$

and the set of linear parameters $\left\{A_{k}\right\}$ as well as the set of non-linear parameters $\left\{b_{k}\right\}$ were determined by minimization of the expectation value of the Hamiltonian. In the present work we will not impose any restriction on $f(r)$ other than the normalizability of the wavefunction.

Due to the linear dependence of the wavefunction on the pair correlation function $f(r)$, the energy functional,

$$
E[f]=\int \Psi^{\dagger} H \Psi \mathrm{d} r_{1} \ldots \mathrm{d} r_{A}
$$

and the norm functional,

$$
N[f]=\int \Psi^{\dagger} \Psi \mathrm{d} r_{1} \ldots \mathrm{d} r_{A}
$$

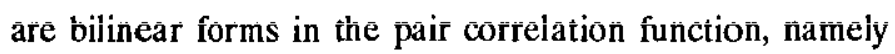

$$
E[f]=\int_{0}^{\infty} \mathrm{d} s \int_{0}^{\infty} \mathrm{d} t f^{*}(s) \boldsymbol{H}(s, t) f(t)
$$

and

$$
N[f]=\int_{\overline{0}}^{\infty} \mathrm{d} s \int_{0}^{\infty} \mathrm{d} t f^{*}(s) N(s, t) f(t) .
$$

To proceed, the two kernels $\boldsymbol{H}(s, t)$ and $N(s, t)$ must be determined. Once these quantities are known, the pair correlation function is obtained by the restricted variational condition

$$
\frac{\delta}{\delta f^{*}}(E[f]-\lambda N[f])=0
$$

where $\lambda$ is the Lagrange multiplier introduced to take into account the normalizability condition of the wavefunction. Its physical values correspond to the eigenenergies of the physical states.

By using the explicit forms of the functionals given by equations (13) and (14), we obtain the linear generalized eigenvalue problem

$$
\int_{0}^{\infty} \mathrm{d} t[\boldsymbol{H}(s, t)-\lambda \bar{N}(s, t)] f(t)=0
$$

which is actually an integro-differential equation. Thus, the two remaining tasks are the determination of the two kernels and the subsequent solution of the eigenvalue problem. 


\section{The norm and potential energy kernels}

This section is devoted to the determination of the kernels corresnonding to the norm and to the potential energy part of the Hamiltonian for the case of a boson system with an arbitrary number of particles $A$. From the point of view of nuclear physics, the only systems which may be considered as bosonic are three- and four-particle systems with nucleons interacting only via Wigner forces, and for specific spin and isospin assignments of each of the individual nucleons. Certainly these systems may not be considered as real models of the physical nuclei ${ }^{3} \mathrm{H},{ }^{3} \mathrm{He}$ or ${ }^{4} \mathrm{He}$, in so far as the interaction considered is spin- and isospin-independent. However, the study of this simplified case is of deep relevance with regard to comparison with such other theories as the Jastrow method [19-21], Faddeev-Yakubovsky techniques [22], approaches using hyperspherical harmonics [23-27], the standard (generalized) shellmodel or configuration-interaction method [8-10], full coupled cluster theory [28], the ATMS method $[29,30]$, the coupled rearrangement method [31] and the Green function Monte Carlo method [32]. Each of these theories has been applied deeply (or is being applied) to these kinds of Wigner-type nuclei. Moreover, the formalism developed here can also be applied to real bosonic systems with an arbitrary, but finite, number of constituents, like the (not yet observed) drops of quantum liquids at very low temperature.

The present formalism is being extended to fermion systems, and to spin- and isospin-dependent interactions. The extension implies some non-trivial technical developments related to the operatorial character of the interaction and of the pair correlations, as well as to the presence of Pauli correlations. Discussion of such extensions of our method is deferred to later work.

For present purposes it is convenient to deal with non-normalized single-particle states corresponding to the lowest harmonic oscillator eigenfunction. The uncorrelated wavefunction (cf equation (9)) will be

$$
\Phi\left(r_{1}, \ldots, r_{A}\right)=\prod_{i=1}^{A} \exp \left(-\alpha^{2} r_{i}^{2} / 2\right)
$$

where $\alpha$ is the inverse length parameter of the harmonic oscillator.

To determine the structure of the kernels it is very useful to employ a diagrammatic representation of the equations. In all cases we may use the simplification of counting only one particular correlation pair in the bra wavefunction, for example $f^{*}\left(r_{12}\right)$. The symmetry of both the interaction Hamiltonian and the ket wavefunction will then compensate for this particular election of the bra. Moreover, this will render quite trivial the caiculation of the functional derivatives $\delta E / \delta f^{*}$ and $\delta N / \delta f^{*}$, which actually correspond to the removal of both the function $f^{*}\left(r_{12}\right)$ in the resulting formulae and the corresponding integration over the coordinate $r_{12}$.

For the diagrammatic representation we will denote the external points $\boldsymbol{r}_{1}$ and $\boldsymbol{r}_{2}$ by means of two full circles, and all other points linked to any correlation operator or any interaction term by means of open circles. The correlation function $f^{*}\left(r_{12}\right)$ is represented by a continuous line connecting the two external points $r_{1}$ and $r_{2}$. The correlation function $f\left(r_{i j}\right)$ coming from the ket wavefunction will also be represented by a continuous line. We note that there is no risk of confusing $f\left(r_{i j}\right)$ lines with the $f^{*}\left(r_{12}\right)$ line. Finally, the two-body interaction will be represented by a broken line connecting the corresponding pair of particles. These diagrams do not include 
explicitly the terms corresponding to the square of the single-particle wavefunction, arising from the uncorrelated state $\Phi\left(r_{1}, \ldots, r_{A}\right)$, and which must be attached to all $A$ particles of the system. This should be taken into account when writing down the equations corresponding to the diagrams.

There is a total of $\left(\begin{array}{c}A \\ 2\end{array}\right)=A(A-1) / 2$ diagrams corresponding to the norm. All these diagrams may be classified into only three topologically distinct structures. These diagrams are shown in figure 1, along with the associated statistical weights.
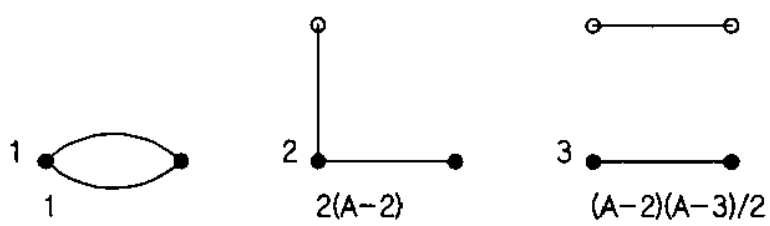

Figure 1. Diagramatic representation of the normalization. Full circles correspond to non-integrated coordinates $r_{1}$ and $r_{2}$, and open circles correspond to other particles, of irrelevant label, to be integrated. There is always a continuous line connecting $\boldsymbol{r}_{1}$ and $\boldsymbol{r}_{2}$ which corresponds to $f^{*}\left(r_{12}\right)$, and another continuous line connecting another two points (say $i$ and $j$ ) and corresponding to $f\left(r_{i j}\right)$. The statistical weight of each diagram is shown in the figure.

The number of diagrams corresponding to the expectation value of the two-body potential (or the two-particle distribution function) is exactly the square of the number of diagrams corresponding to the norm. They are counted by allowing for a two-body interaction on any pair of particles, as well as any (right-hand side) pair correlation function between any pair of particles, in addition to the $f^{*}\left(r_{12}\right)$ pair correlation. All of these diagrams may be classified into the 16 different topological structures shown in figure 2, again with their corresponding statistical weights. In order to clarify the meaning of this diagrammatic notation we write down the integral corresponding to diagram 6 of figure 2,

$V_{\varepsilon}=\int \mathrm{d} r_{1} \mathrm{~d} r_{2} \ldots \mathrm{d} r_{A} f^{*}\left(r_{12}\right) V\left(r_{24}\right) f\left(r_{13}\right) \exp \left(-\alpha^{2} \sum_{i} r_{i}^{2}\right)$.

To facilitate the calculation of the functional derivatives required by the EulerLagrange variational principle, all integrals corresponding to these diagrams have to be simplified so that they involve explicitly an integral on the variable $\boldsymbol{r}_{12}$ and also on the corresponding coordinate associated with the other pair correlation $\boldsymbol{r}_{i j}$. Clearly, all analytically evaluable integrals should also be carried out. The first step is thus to integrate over all particles which do not appear in the diagrams and which carry only the squared single-particle harmonic-oscillator wavefunction. Such integrals are trivially performed using the relation

$$
I(\alpha)=\int \exp \left(-\alpha^{2} r^{2}\right) \mathrm{d} \boldsymbol{r}=\left(\pi / \alpha^{2}\right)^{3 / 2} .
$$

The second step is to observe that unlinked diagrams may be split into their disconnected pieces, in such a way that the integrals to be further reduced will correspond only to the generic forms represented by the diagrams 1, 2, 5, 7 and 9 

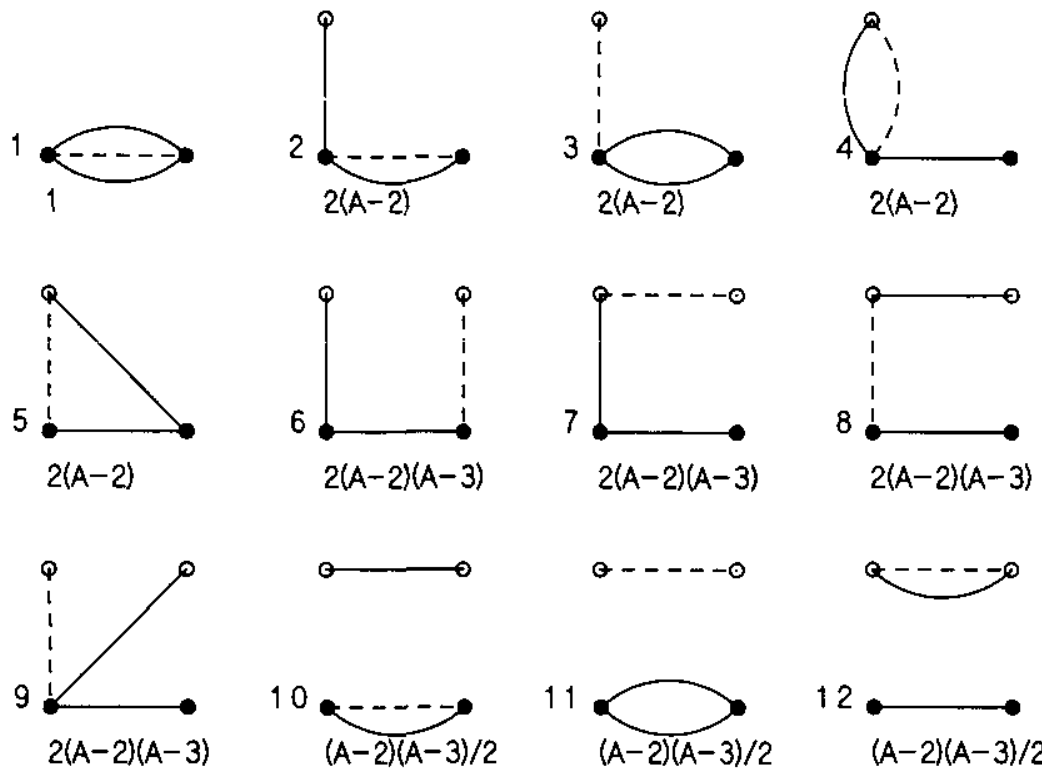

12 $(A-2)(A-3) / 2$
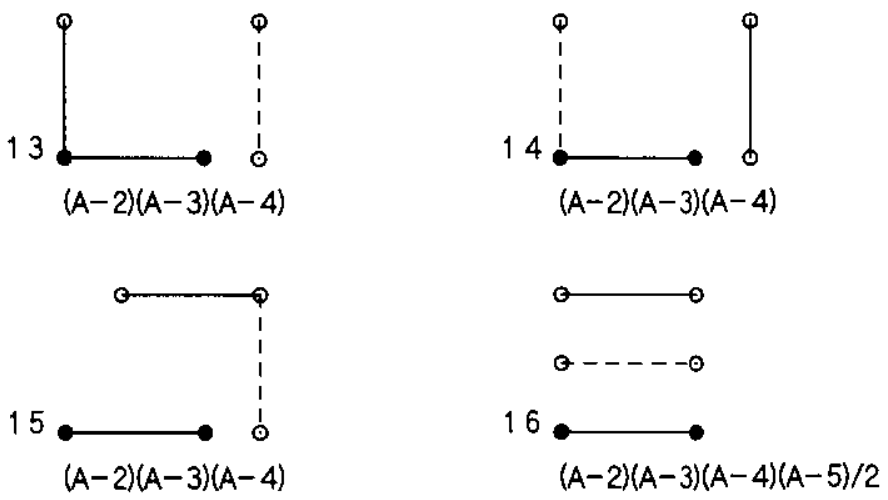

Figure 2. Diagrammatic representation of the expectation value of the two-body interaction potential. In addition to the specifications of figure 1, there appears a new object, a broken line, which corresponds to the two-body potential $V\left(r_{i j}\right)$ between particles $i$ and $j$.

of figure 2. These generic diagrams require special coordinate transformations for their maximal simplification. The appropriate transformations are described in detail in the appendix.

We now show in detail expressions for all distinct forms contributing to the norm and potential energy kernels. Because of some important simplifications related to the structure of the kernel corresponding to the kinetic energy, it turns out to be convenient to define a new pair correlation function

$$
\phi(r)=r f(r) \exp \left(-\alpha^{2} r^{2} / 4\right)
$$

which absorbs the terms coming from the Jacobian of the transformation from Cartesian coordinates to spherical coordinates as well as the integration measure associated 
with the single-particle wavefunctions. As will be shown in the next section, with this substitution the kernel corresponding to the kinetic energy turns out to be symmetric, thereby guaranteeing the reality of the eigenvalues.

We thus rewrite (14) and (13) in the equivalent forms

$$
N[f]=\int_{0}^{\infty} \mathrm{d} s \int_{0}^{\infty} \mathrm{d} t \phi^{*}(s) \mathcal{N}(s, t) \phi(t)
$$

and

$$
E[f]=\int_{0}^{\infty} \mathrm{d} s \int_{0}^{\infty} \mathrm{d} t \phi^{*}(s)[\mathcal{K}(s, t)+\mathcal{V}(s, t)] \phi(t)
$$

with kinetic and potential energy kernels $\mathcal{K}(s, t)$ and $\mathcal{V}(s, t)$ respectively.

The contributions to the norm kernel from the three terms represented pictorially in figure 1 are, respectively,

$$
\begin{aligned}
& \mathcal{N}_{1}=4 \pi I^{A-2}(\alpha) I(\sqrt{2} \alpha) \delta(s-t) \\
& \mathcal{N}_{2}=48 \pi^{2} \alpha^{-2}(A-2) I^{A-3}(\alpha) I(\sqrt{3} \alpha) \sinh \left(2 \alpha^{2} s t / 3\right) \\
& \quad \times \exp \left(-5 \alpha^{2}\left(s^{2}+t^{2}\right) / 12\right) \\
& \mathcal{N}_{3}=8 \pi^{2}(A-2)(A-3) I^{A-4}(\alpha) I^{2}(\sqrt{2} \alpha) s t \exp \left(-\alpha^{2}\left(s^{2}+t^{2}\right) / 4\right) .
\end{aligned}
$$

Using again the procedure described in the appendix one may obtain the following set of equations corresponding to the potential energy kernel $\mathcal{V}$, which has been divided into parts according to the diagrammatic representation of figure 2 :

$$
\begin{aligned}
& \mathcal{V}_{1}=4 \pi I^{A-2}(\alpha) I(\sqrt{2} \alpha) V(s) \delta(s-t) \\
& V_{2}+V_{4}=48 \pi^{2}(A-2) I^{A-3}(\alpha) I(\sqrt{3} \alpha) \alpha^{-2} \exp \left(-5 \alpha^{2}\left(s^{2}+t^{2}\right) / 12\right) \\
& \quad \times \sinh \left(2 \alpha^{2} s t / 3\right)[V(s)+V(t)] \\
& V_{3}=48 \pi^{2}(A-2) I^{A-3}(\alpha) I(\sqrt{3} \alpha) \alpha^{-2} s^{-1} \exp \left(-\alpha^{2} s^{2} / 6\right) \delta(s-t) \\
& \quad \times \int_{0}^{\infty} u \mathrm{~d} u V(u) \exp \left(-2 \alpha^{2} u^{2} / 3\right) \sinh \left(2 \alpha^{2} s u / 3\right) \\
& \mathcal{V}_{5}=16 \pi^{2}(A-2) I^{A-3}(\alpha) I(\sqrt{3} \alpha) \exp \left(-\alpha^{2}\left(s^{2}+t^{2}\right) / 12\right) \\
& \times \int_{|s-t|}^{s+t} u \mathrm{~d} u V(u) \exp \left(-\alpha^{2} u^{2} / 3\right) \\
& V_{6}=128 \pi^{3}(A-2)(A-3) I^{A-4}(\alpha) I(2 \alpha) s t \exp \left(-\alpha^{2}\left(2 t^{2}+3 s^{2}\right) / 4\right) \\
& \times \int_{0}^{\infty} u^{2} \mathrm{~d} u V(u) \exp \left(-3 \alpha^{2} u^{2} / 4\right) \\
& \times \sum_{n}(-1)^{n}(2 n+1) i_{n}\left(\alpha^{2} s t\right) i_{n}\left(\alpha^{2} s u\right) i_{n}\left(\alpha^{2} t u / 2\right) \\
& V_{7}=128 \pi^{3}(A-2)(A-3) I^{A-4}(\alpha) I(2 \alpha) s t \exp \left(-\alpha^{2}\left(2 s^{2}+3 t^{2}\right) / 4\right)
\end{aligned}
$$




$$
\begin{aligned}
& \times \int_{0}^{\infty} u^{2} \mathrm{~d} u V(u) \exp \left(-3 \alpha^{2} u^{2} / 4\right) \\
& \times \sum_{n}(-1)^{\bar{\pi}}(2 n+1) i_{n}\left(\alpha^{2} s t\right) i_{n}\left(\alpha^{2} t u\right) i_{n}\left(\alpha^{2} s u / 2\right)
\end{aligned}
$$

$$
\mathcal{V}_{8}=128 \pi^{3}(A-2)(A-3) I^{A-4}(\alpha) I(2 \alpha) s t \exp \left(-\alpha^{2}\left(s^{2}+t^{2}\right) / 2\right)
$$

$$
\begin{aligned}
& \times \int_{0}^{\infty} u^{2} \mathrm{~d} u V(u) \exp \left(-\alpha^{2} u^{2}\right) \\
& \times \sum_{n}(-1)^{n}(2 n+1) i_{n}\left(\alpha^{2} s t / 2\right) i_{n}\left(\alpha^{2} s u\right) i_{n}\left(\alpha^{2} t u\right)
\end{aligned}
$$

$$
\mathcal{V}_{9}=128 \pi^{3}(A-2)(A-3) I^{A-4}(\alpha) I(2 \alpha) s t \exp \left(-\alpha^{2}\left(s^{2}+t^{2}\right) / 2\right)
$$

$$
\begin{aligned}
& \times \int_{0}^{\infty} u^{2} \mathrm{~d} u V(u) \exp \left(-\alpha^{2} u^{2} / 2\right) \\
& \times \sum_{n}(2 n+1) i_{n}\left(\alpha^{2} s t / 2\right) i_{n}\left(\alpha^{2} s u / 2\right) i_{n}\left(\alpha^{2} t u / 2\right)
\end{aligned}
$$

$$
\begin{aligned}
\mathcal{V}_{10}+\mathcal{V}_{12}= & 8 \pi^{2}(A-2)(A-3) I^{A-4}(\alpha) I^{2}(\sqrt{2} \alpha) s t \\
& \times \exp \left(-\alpha^{2}\left(s^{2}+t^{2}\right) / 4\right)[V(s)+V(t)]
\end{aligned}
$$$$
\mathcal{V}_{11}=8 \pi^{2}(A-2)(A-3) I^{A-4}(\alpha) I^{2}(\sqrt{2} \alpha) \delta(s-t) \int_{0}^{\infty} u^{2} d u \exp \left(-\alpha^{2} u^{2} / 2\right) V(u)
$$$$
\mathcal{V}_{13}=96 \pi^{3}(A-2)(A-3)(A-4) I^{A-5}(\alpha) I(\sqrt{2} \alpha) I(\sqrt{3} \alpha) \alpha^{-2}
$$$$
\times \exp \left(-5 \alpha^{2}\left(s^{2}+t^{2}\right) / 12\right) \sinh \left(2 \alpha^{2} s t / 3\right)
$$$$
\times \int_{0}^{\infty} u^{2} \mathrm{~d} u V(u) \exp \left(-\alpha^{2} u^{2} / 2\right)
$$

$$
\mathcal{V}_{14}=96 \pi^{3}(A-2)(A-3)(A-4) I^{A-5}(\alpha) I(\sqrt{2} \alpha) I(\sqrt{3} \alpha) \alpha^{-2} t
$$$$
\times \exp \left(-\alpha^{2} t^{2} / 4-5 \alpha^{2} s^{2} / 12\right)
$$$$
\times \int_{0}^{\infty} u \mathrm{~d} u V(u) \exp \left(-2 \alpha^{2} u^{2} / 3\right) \sinh \left(2 \alpha^{2} s u / 3\right)
$$

$$
\mathcal{V}_{15}=96 \pi^{3}(A-2)(A-3)(A-4) I^{A-5}(\alpha) I(\sqrt{2} \alpha) I(\sqrt{3} \alpha) \alpha^{-2} s
$$

$$
\times \exp \left(-\alpha^{2} s^{2} / 4-5 \alpha^{2} t^{2} / 12\right)
$$$$
\times \int_{0}^{\infty} u \mathrm{~d} u V(u) \exp \left(-2 \alpha^{2} u^{2} / 3\right) \sinh \left(2 \alpha^{2} t u / 3\right)
$$

$$
\mathcal{V}_{16}=16 \pi^{3}(A-2)(A-3)(A-4)(A-5) I^{A-6}(\alpha) I^{3}(\sqrt{2} \alpha) s t
$$

$$
\times \exp \left(-\alpha^{2}\left(s^{2}+t^{2}\right) / 4\right) \int_{0}^{\infty} u^{2} \mathrm{~d} u V(u) \exp \left(-\alpha^{2} u^{2} / 2\right)
$$

where $i_{n}$ is the modified Bessel function, as defined in the appendix. 


\section{The reduction of the kinetic energy kernel}

To obtain the kernel associated with the kinetic energy operator it is convenient to carry out some prior algebraic transformations. The action of the kinetic energy operator on the ket wavefunction is

$$
\sum_{n} \nabla_{n}^{2} \sum_{i<j} f\left(r_{i j}\right) \exp \left(-\alpha^{2} \sum_{k} r_{k}^{2} / 2\right)=K^{(1)}+K^{(2)}+K^{(3)}
$$

with

$$
\begin{aligned}
& K^{(1)}=2 \sum_{i<j}\left(f^{\prime \prime}\left(r_{i j}\right)+\frac{2}{r_{i j}} f^{\prime}\left(r_{i j}\right)-\alpha^{2} r_{i j} f^{\prime}\left(r_{i j}\right)\right) \exp \left(-\alpha^{2} \sum_{k} r_{k}^{2} / 2\right) \\
& K^{(2)}=-3 A \alpha^{2} \sum_{i<j} f\left(r_{i j}\right) \exp \left(-\alpha^{2} \sum_{k} r_{k}^{2} / 2\right)
\end{aligned}
$$

and

$$
K^{(3)}=\alpha^{4} \sum_{n} r_{n}^{2} \sum_{i<j} f\left(r_{i j}\right) \exp \left(-\alpha^{2} \sum_{k} r_{k}^{2} / 2\right) .
$$

As we have already mentioned in the preceding section, the first term of the right-hand side of (25) is simplified after changing the pair correlation function $f(r)$ to a new one $\phi(r)$,

$$
f(r)=\frac{1}{r} \phi(r) \exp \left(\frac{\alpha^{2} r^{2}}{4}\right)
$$

producing

$$
\begin{aligned}
K^{(1)}=\sum_{i<j} & {\left[\left(\frac{2}{r_{i j}} \phi^{\prime \prime}\left(r_{i j}\right)+\frac{3 \alpha^{2}}{r_{i j}} \phi\left(r_{i j}\right)-\frac{\alpha^{4}}{2} r_{i j} \phi\left(r_{i j}\right)\right) \exp \left(\frac{\alpha^{2} r_{i j}^{2}}{4}\right)\right] } \\
& \times \exp \left(-\alpha^{2} \sum_{k} \frac{r_{k}^{2}}{2}\right) .
\end{aligned}
$$

The kernel associated with this part is then formally equivalent to the normalization kernel, after replacing the ket pair correlation function by the quantity appearing in the square brackets of (30), and including the overall constant $-\hbar^{2} / 2 m$.

We thus obtain for this contribution to the energy the value

$$
\mathcal{K}^{(1)}=\left(\frac{-\hbar^{2}}{2 m}\right)\left[\mathcal{N}_{1}+\mathcal{N}_{2}+\mathcal{N}_{3}\right]\left(2 D_{t}^{2}+3 \alpha^{2}-\frac{\alpha^{4} t^{2}}{2}\right)
$$

where $D_{t}^{2}$ is the second derivative operator with respect to the coordinate $t$. It acts only on the pair correlation function $\phi(t)$. This expression should be symmetrized by interchanging the term in square brackets with the term in parentheses, and 
substituting in the latter $t$ by $s$, i.e. considering the derivative operator acting on the bra pair correlation function $\phi^{*}(s)$. The two terms are then averaged, thus restoring the symmetry of the kernel. This averaging process will not affect the fina! value of the integral, as it only involves the addition of a zero-measure surface term.

The second term on the right-hand side of equation (25) is trivially evaluated, being proportional to the norm:

$$
\mathcal{K}^{(2)}=3 A \alpha^{2} \hbar^{2} / 2 m\left[\mathcal{N}_{1}+\mathcal{N}_{2}+\mathcal{N}_{3}\right]
$$

Finally the third term may be obtained in the following indirect form. By computing the derivative of the norm with respect to $\alpha^{2}$ we find

$$
\frac{\mathrm{d} N}{\mathrm{~d} \alpha^{2}}=-\int \mathrm{d} \boldsymbol{r}_{1} \mathrm{~d} \boldsymbol{r}_{2} \ldots \mathrm{d} \boldsymbol{r}_{A} f^{*}\left(r_{12}\right) \sum_{i<j} f\left(r_{i j}\right) \sum_{n} r_{n}^{2} \exp \left(-\alpha^{2} \sum_{k} r_{k}^{2}\right)
$$

which, apart from a constant, is the quantity $K^{(3)}$. The evaluation of this derivative is quite tedious. The values corresponding to this part of the kinetic energy arising from the three terms of (23) are, respectively,

$$
\begin{aligned}
& \mathcal{K}_{1}^{(3)}=\left(-\hbar^{2} / 2 m\right) \pi I^{A-2}(\alpha) I(\sqrt{2} \alpha) \alpha^{2}\left(6(A-1)+2 \alpha^{2} s^{2}\right) \delta(s-t) \\
& \mathcal{K}_{2}^{(3)}=\left(-\hbar^{2} / 2 m\right) 8 \pi^{2}(A-2) I^{A-3}(\alpha) I(\sqrt{3} \alpha) \exp \left(-5 \alpha^{2}\left(s^{2}+t^{2}\right) / 12\right) \\
& \times\left[\sinh \left(2 \alpha^{2} s t / 3\right)\left[9 A-12+4 \alpha^{2}\left(s^{2}+t^{2}\right)\right]\right. \\
& \left.-4 \alpha^{2} s t \cosh \left(2 \alpha^{2} s t / 3\right)\right] \\
& \mathcal{K}_{\overline{3}}^{(3)}=\left(-\hbar^{2} / 2 m\right) 4 \pi^{2}(A-2)(A-3) I^{A-4}(\alpha) I^{2}(\sqrt{2} \alpha) \alpha^{2} s t \\
& \times \exp \left(-\alpha^{2}\left(s^{2}+t^{2}\right) / 4\right)\left[3 A-6+\alpha^{2}\left(s^{2}+t^{2}\right)\right] \text {. }
\end{aligned}
$$

\section{The numerical solution of the eigenvalue problem}

In this section we describe the solution of the generalized eigenvalue problem of equation (16) corresponding to the kernel obtained in the two previous sections. This is actually an integro-differential equation, analogous to the Schrödinger equation, but with a non-local potential. Terms like $\delta(s-t) D_{t}^{2}$ coming from the kinetic energy part of the kernel, when introduced in equation (16), give rise to a second derivative of the pair correlation function. All terms which contain the delta function are local contributions to the effective potential, and the remaining terms are non-local contributions.

Instead of attempting a step-by-step solution of this integro-differential equation, it seems simpler to reformulate the probem in terms of a global matrix diagonalization procedure. Thus we simply assume a grid with step $h$ in which both $s$ and $t$ are discretized, so that in correspondence with $\mathcal{H}(s, t)$ there is a square matrix $\mathcal{H}_{i j}$, where $s=i h$ and $t=j h$. All explicit integrals are replaced by the trapezoidal rule,

$$
\int_{0}^{\infty} \mathrm{d} t \mathcal{H}(s, t) \phi(t) \rightarrow h \sum_{j=1}^{N} \mathcal{H}_{i, j} \phi_{j}
$$


where $N h=s_{\max }=t_{\max }$ is an upper boundary for both $s$ and $t$ which replaces the upper integration limit of infinity. There are two special cases, namely the delta function and the second derivative operator, which merit further discussion. The delta function is replaced by a diagonal matrix

$$
\delta(s-t) \rightarrow \delta_{i j} / h
$$

and the second derivative operator is replaced by a tri-diagonal matrix corresponding to the second differences operator

$$
D^{2} g(x) \rightarrow(g(x-h)-2 g(x)+g(x+h)) / h^{2} .
$$

The matrix is finite because of the boundary conditions and the structure of the transformed pair correlation function of equation (20). Given that $\phi(0)=0$, the origin does not explicitly enter in the construction of the matrix (and hence the lower limit for the sum over $j$ in equation (35) is 1 ). On the other hand, $\phi(s)$ tends to zero for large values of $s$, so one may introduce an $s_{\max }$ beyond which the eigenvector is effectively null.

The numerical approximation has an error $\mathrm{O}\left(h^{2}\right)$, so that a sufficiently small integration step has to be used in order to get reliable results. The precision may be noticeably increased by means of the well known Richardson extrapolation algorithm [33]. It consists of extrapolating the results corresponding to a given step $h$ and another step $2 h$ to $h=0$, and results in an $\mathrm{O}\left(h^{4}\right)$ algorithm. In our case, we found that a mesh of 160 points extending up to $10 \mathrm{fm}$ gave sufficient precision.

It is also worth mentioning the technique used to evaluate the infinite sums involving the modified spherical Bessel functions $i_{n}(x)$. For a given value of $x$ there is always a value of $n$ at which $\dot{i}_{n}(x) \ll 1$ and $\dot{i}_{n+1}(x) \ll \dot{x}_{n}(x)$. This permits the use of a backwards recurrence relation (which will be stable against roundoff) with the initial values $i_{n+1}(x)=0$ and $i_{n}(x)=1$, and a final renormalization of all values against the known value of $i_{0}(x)=\sinh (x) / x$. In our case it was necessary to compute up to 40 Bessel functions.

With the techniques described, the precision attained in the lowest eigenvalue was found to be of 1 part in $10^{8}$, by comparison with a comparable calculation using a correlation function parametrized as in equation (10).

\section{Results and conclusions}

The previous formalism has been applied to the evaluation of the ground-state energy of the ${ }^{4} \mathrm{He}$ nucleus and using as a two-body interaction both the Malfliet-Tjon MTV force [13] (with the Zabolitzky parametrization [32]) and the spin-averaged $\$ 3$ interaction of Afnan and Tang [12]. These two interactions are fitted to the low-energy phase shifts in the two-nucleon even channels, so that they may be considered as realistic. Both are soft-core interactions, with a yukawian shape in the case of MTV and a finite core in the case of $S 3$.

Figure 3 represents the values of the GS energy for the MTV force as a function of the variational parameter $\alpha$, corresponding to the harmonic oscillator uncorrelated state. This figure includes the shell-model-like calculations which describe the pair correlation function as a mixture of translationally invariant $2 \mathrm{p}-2 \mathrm{~h}$ operators as in 


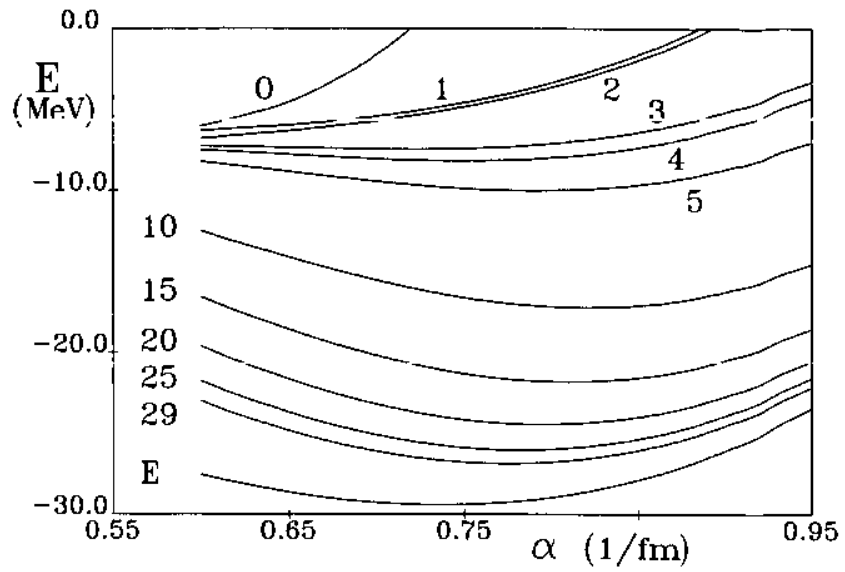

Figure 3. The Gs energy in $\mathrm{MeV}$ of ${ }^{4} \mathrm{He}$ corresponding to the MTV interaction versus the harmonic oscillator parameter $\alpha$. The lines labelled with an integer number $n_{\max }$ represent the shell-model calculation with $2 \mathrm{p}-2 \mathrm{~h}$ excitations up to $2 n_{\max } \hbar \omega$ in energy. The curve labelled $E$ is the present Euler-Lagrange evaluation of this energy.

equation (4), but where the infinite upper limit of the sum $n$ is replaced by some $n_{\max }$. We have considered subspaces of increasing dimensionality up to $n_{\max }=29$, which corresponds to 30 states, or equivalently up to $58 \hbar \omega$ of excitation energy of the corresponding pair of particles. We also show the present Euler-Lagrange determination (line labelied E), which is the limit of the previous shell-model calculations for an infinite number of $2 \mathrm{p}-2 \mathrm{~h}$ excitations $\left(n_{\max } \rightarrow \infty\right)$. The figure actually shows that the shell-model-like expansion approaches the limiting value very slowly, particularly when dealing with strongly repulsive interactions at short distances, as we had already guessed in our previous work $[1,7]$.

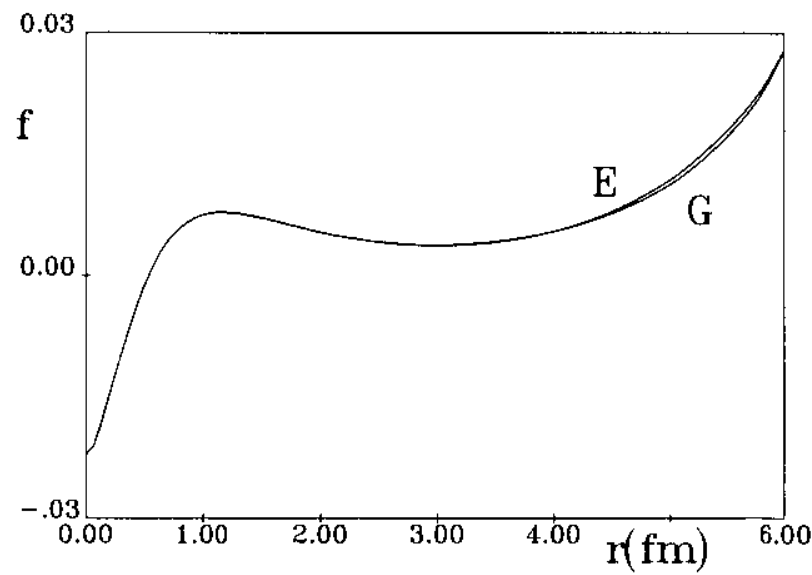

Figure 4. Comparison of the pair correlation function $f(r)$ (in arbitrary units) computed with the Euler-Lagrange integro-differential equations (E) and with the Gaussian geminal expansion of equation (10) employing eight terms in the sum (G) for the MTV interaction potential.

This figure does not include the values of the energy which result from the Gaus- 
sian expansion of the pair correlation amplitude [14], since these are indistinguishable from those found by the present optimal Euler-Lagrange technique. In fact, there is a gain of only $0.04 \mathrm{MeV}$ in the present Euler-Lagrange description as compared with the expansion of equation (10) including only eight terms. There is, however, a very small difference between the corresponding pair correlation functions, as shown in figure 4 , but even this occurs only at rather long distances. This small difference, which has no sizeable effects on the energy, is a consequence of the different behaviour of the two descriptions (Gaussian representation and coordinate-space representation) at long distances. However, we should bear in mind that the effective pair correlation function is, in fact, the product of $f(r)$ times a rapidly decreasing function $r \exp \left(-\alpha^{2} r^{2} / 4\right)$, as in equation (20), so that the effective correlation is null at such long distances. Thus we may conclude that for the present calculation there is no need to go to this cumbersome Euler-Lagrange approach, as the much simpler Gaussian expansion employing relatively few terms is able to produce almost the same result. Nevertheless, the situation may be very different when dealing with state-dependent pair correlations, as one should do in realistic descriptions of light nuclei. In such a case the number of variational parameters in the Gaussian expansion, with respect to which minimization must be performed, may be so large that it may actually be simpler to solve the set of coupled equations produced by the Euler-Lagrange approach.

Table 1. Comparison of the results obtained with the present TiC[ $\left.C_{2}\right]-\mathrm{EL}$ optimal EulerLagrange approach with closely related theories for the interactions MTV and S3. The values correspond to the GS energy in MeV for the two poientials.

\begin{tabular}{lll}
\hline & \multicolumn{2}{c}{ Potential } \\
\cline { 2 - 3 } Method & MTV & $\mathrm{S} 3$ \\
\hline $\mathrm{TC}\left[C_{2}\right]-\mathrm{EL}$ & -29.43 & -25.37 \\
$\mathrm{PPB}^{\mathrm{a}, \mathrm{b}}$ & -30.05 & -26.005 \\
$\mathrm{CPB}^{\mathrm{C}}$ & -31.344 & -27.369 \\
DDEA $^{\mathrm{d}}$ & -30.68 & -27.09 \\
$\mathrm{SM}^{\mathrm{C}}$ & -18.31 & - \\
$\left.\mathrm{TC} C C_{3}^{\mathrm{F}}+C_{2}^{(2)}\right]^{\mathrm{r}}$ & -31.24 & -27.21 \\
$\mathrm{ATMS}^{\mathrm{g}}$ & -31.36 & - \\
$\mathrm{DMC}^{\mathrm{h}}$ & $-31.32 \pm 0.02$ & $-27.35 \pm 0.02$ \\
\hline
\end{tabular}

a Hyperspherical harmonics description in the potential basis approximation with pair correlations [27].

b [35].

c Jastrow correlated hyperspherical harmonics description [34].

d Integro-differential equation approach to the hyperspherical harmonics expansion [24].

- Shell-model calculation in the full $10 \hbar \omega$ space [9].

f Translationally invariant cluster calculation including two- and three-body clusters as well as quadratic two-body clusters [15].

8 The arms method [30].

h Diffusion Monte Carlo calculation witls importance sampling [36].

In table 1 we compare our results with other calculations employing the realistic potentials MTV and S3 for the ${ }^{4} \mathrm{He}$ nucleus. We have included results for the hyperspherical harmonics description (including pair correlations or Jastrow correlations), the shell-model description, the coupled cluster theory and the ATMS method. All of these methods have many things in common with our simple linearized S2 calculation. 
As we have already commented earlier, the shell-model (SM) calculation [9] considers all possible states with excitation energy up to $10 \hbar \omega$ (this corresponds to a basis of 2765 states), and the energy obtained is still very far from the exact (within statistical errors) results of the diffusion Monte Carlo method (DMC). Undoubtedly, if a sufficiently large number of states were included in the SM basis, the exact result would have been obtained, but this seems an impracticable task.

The methods based on the hyperspherical harmonics expansion have a particular similarity with our own approach. The wavefunction is of the form

$$
\Psi=\sum_{i<j} g\left(r_{i j}, R\right)
$$

where $R$ is the hyper-radius of the four-particle system

$$
R^{2}=\sum_{i<j} r_{i j}^{2}
$$

The system is thus described in terms of pair correlations which depend mainly on the interparticle distance $r_{i j}$, but which also depend on the hyper-radius of the full system. In the approximation called PPB (pair-correlated potential basis) in table 1, the pair correlation function is factorized into two terms, one depending only on $r_{i j}$ and the other on $R$. The latter is then expanded in a sub-basis of the full hyperspherical harmonic basis (namely, the so called potential basis) and the energy is determined variationally. The true pair correlation term is maintained fixed, and determined through the solution of a screened Schrödinger equation for the pair of particles. This procedure is almost the opposite of what we are doing. Thus, we maintain fixed the part depending on the hyper-radius (the $(0 s)^{4}$ harmonic oscillator state is the first state of the hyperspherical basis) and concentrate all our effort into improving the pair correlation function.

The approximation called CPB [34] is a generalization of the PPB approach. Instead of including only correlations between independent pairs of particles, all of them are correlated by means of a Jastrow ansatz. Finally, the integro-differential equation approach (IDEA) approximates equation (38) so as to get coupled integro-differential equations relating the pair correlation part and the hyper-radius-dependent part. Because of the approximations involved the variational character is, however, lost. Each of these methods represents an improvement in principle on our approach. Nevertheless, as we observe from table 1, each of these improvements results in a relatively small change in the GS energy. We should mention that the CPB approach is an almost exact description of the system, but it requires the evaluation of multidimensional integrals. Finally, in table 1 we have also included our own systematic improvement of the translationally invariant cluster description, in the approximation termed $\operatorname{TrC}\left[C_{3}^{\mathrm{F}}+C_{2}^{(2)}\right]$, described in detail in [15]. Basically, we now incorporate all twoand three-body correlations, together with the quadratic pair correlations. This later calculation was performed by expanding both the pair and the triplet correlation functions in terms of Gaussian geminal bases. As we have already stated, one of the primary aims of the present calculation has been to verify the high accuracy attainable by relatively small such bases at the $\mathrm{nC}\left[C_{2}\right]$ level, by comparison with the optimal variational calculation at the same level reported herein. The results of the extension to include the extra terms in the $\operatorname{TIC}\left[C_{3}^{\mathrm{F}}+C_{2}^{(2)}\right]$ approximation are thereby 
confidently expected to lie very close to the minimum attainable in this expanded correlation subspace.

The values obtained for the GS energy by using the $\mathrm{CPB}$ and the $\operatorname{TIC}\left[C_{3}^{\mathrm{F}}+C_{2}^{(2)}\right]$ methods cannot be distinguished from the Monte Carlo results, indicating the importance of the three-body correlations (which produce the last 1-2 MeV of binding energy) in addition to the pair correlations which comprise the bulk of the binding energy.

\section{Acknowledgments}

EB and RG are supported by the Dirección General de Investigación Científica y Tecnológica, Spain. MFF acknowledges support via a fellowship from the Ministerio de Educación y Ciencia, Spain, under the programme Estancias Temporales de Cientificos y Tecnólogos Extranjeros. RFB acknowledges the support of a research grant from the Science and Engineering Research Council (SERC) of Great Britain.

\section{Appendix. The reduction of the functional integrals}

The simplest case corresponds to the diagram 1 of figure 2. Once the integrations on $r_{3}, r_{4}, \ldots$, up to $r_{A}$ are performed, giving the contribution $I^{A-2}(\alpha)$, there remains the contribution

$$
D_{1}=\int \mathrm{d} r_{1} \mathrm{~d} r_{2} \exp \left(-\alpha^{2}\left(r_{1}^{2}+r_{2}^{2}\right)\right) g\left(r_{12}\right)
$$

where $g\left(r_{12}\right)$ is a generic function to be substituted by the appropriate functions corresponding to the norm or the potential matrix elements. The only possible simplifications with this diagram are to change to center-of-mass and relative coordinates, carry out the centre-of-mass integration and finally evaluate the angular integrations related to the relative coordinate. There remains

$$
D_{1}=4 \pi I(\sqrt{2} \alpha) \int_{0}^{\infty} s^{2} \mathrm{~d} s g(s) \exp \left(-\alpha^{2} s^{2} / 2\right)
$$

The next diagram in order of increasing complexity corresponds to an integral of the form (see diagrams 2,3 and 4 of figure 2)

$$
D_{2}=\int \mathrm{d} \boldsymbol{r}_{1} \mathrm{~d} \boldsymbol{r}_{2} \mathrm{~d} \boldsymbol{r}_{3} \exp \left[-\alpha^{2}\left(r_{1}^{2}+r_{2}^{2}+r_{3}^{2}\right)\right] g\left(r_{12}\right) h\left(r_{13}\right)
$$

which may be reduced by changing to the new coordinates

$$
\begin{aligned}
& \boldsymbol{s}=\boldsymbol{r}_{1}-\boldsymbol{r}_{2} \\
& \boldsymbol{t}=\boldsymbol{r}_{1}-\boldsymbol{r}_{3} \\
& \boldsymbol{R}=\left(\boldsymbol{r}_{1}+\boldsymbol{r}_{2}+r_{3}\right) / 3
\end{aligned}
$$


and integrating over $\boldsymbol{R}$ and all remaining angles, with the result

$$
\begin{aligned}
& D_{2}=21 \pi^{2} \alpha^{-2} I(\sqrt{3} \alpha) \int_{0}^{\infty} s \mathrm{~d} s \int_{0}^{\infty} t \mathrm{~d} t g(s) h(t) \\
& \times \exp \left[-2 \alpha^{2}\left(s^{2}+t^{2}\right) / 3\right] \sinh \left(2 \alpha^{2} s t / 3\right) .
\end{aligned}
$$

With the help of $D_{1}$ and $D_{2}$ one can also compute the contributions of diagrams 10-16 of figure 2, as well as diagram 3 of figure 1 .

The other diagram involving three particles corresponds to the triangle graph (diagram 5 of figure 2), with generic form

$$
D_{5}=\int \mathrm{d} \boldsymbol{r}_{1} \mathrm{~d} \boldsymbol{r}_{2} \mathrm{~d} \boldsymbol{r}_{3} g\left(r_{12}\right) h\left(r_{13}\right) w\left(r_{23}\right) \exp \left[-\alpha^{2}\left(r_{1}^{2}+r_{2}^{2}+r_{3}^{2}\right)\right] .
$$

This is the well known integral over a triangle. The centre-of-mass coordinate of the three particles is easily integrated out, as well as three of the remaining six variables. This is the maximum simplification of the diagram. However, the resulting form is conveniently represented by using as integration variables the three lengths of the sides of the triangle, with the result

$$
\begin{gathered}
D_{5}=8 \pi^{2} I(\sqrt{3} \alpha) \int_{0}^{\infty} s \mathrm{~d} s \int_{0}^{\infty} t \mathrm{~d} t \int_{|s-t|}^{s+t} u \mathrm{~d} u g(s) h(t) w(u) \\
\times \exp \left[-\alpha^{2}\left(s^{2}+t^{2}+u^{2}\right) / 3\right]
\end{gathered}
$$

Finally there remain only irreducible diagrams involving four particles, which are more cumbersome to reduce. Diagrams 6, 7 and 8 of figure 2 have the same structure, and may be generically described by the integral

$$
D_{7}=\int \mathrm{d} \boldsymbol{r}_{1} \mathrm{~d} \boldsymbol{r}_{2} \mathrm{~d} \boldsymbol{r}_{3} \mathrm{~d} \boldsymbol{r}_{4} g\left(r_{12}\right) h\left(r_{13}\right) w\left(r_{34}\right) \exp \left[-\alpha^{2}\left(r_{1}^{2}+r_{2}^{2}+r_{3}^{2}+r_{4}^{2}\right)\right] \text {. }
$$

This form suggests the change of variables

$$
\begin{aligned}
& \boldsymbol{s}=\boldsymbol{r}_{1}-\boldsymbol{r}_{2} \\
& \boldsymbol{t}=\boldsymbol{r}_{1}-\boldsymbol{r}_{3} \\
& \boldsymbol{u}=\boldsymbol{r}_{3}-\boldsymbol{r}_{4} \\
& \boldsymbol{R}=\left(\boldsymbol{r}_{1}+\boldsymbol{r}_{2}+\boldsymbol{r}_{3}+\boldsymbol{r}_{4}\right) / 4 .
\end{aligned}
$$

The integration on $\boldsymbol{R}$ gives rise to the factor $I(2 \alpha)$. For the remaining integrals it turns out to be convenient to go to spherical coordinates. The dependence on the angles appears only in forms like $\exp (z \cos \theta)$, where $\theta$ is one of the angles between two of the vectors $s, t$ and $u$. To carry out the integral over all angular coordinates it is convenient to use the analogue of the well known plane-wave expansion [37]

$$
\exp ( \pm z \cos \theta)=\sum_{n=0}^{\infty}( \pm 1)^{n}(2 n+1) i_{n}(z) P_{n}(\cos \theta)
$$


involving the modified spherical Bessel functions $i_{n}$ (counterparts of the familiar spherical Bessel functions $j_{n}$ ) defined as

$$
i_{n}(z)=\sqrt{\frac{\pi}{2 z}} I_{n+\frac{1}{2}}(z) .
$$

Using this expansion, rewriting the Legendre polynomials in spherical harmonics, and using the orthogonality properties of the the latter leads to the final result

$$
\begin{aligned}
D_{7}=(4 \pi)^{3} I(2 \alpha) \int_{0}^{\infty} s^{2} \mathrm{~d} s \int_{0}^{\infty} t^{2} \mathrm{~d} t \int_{0}^{\infty} u^{2} \mathrm{~d} u g(s) h(t) w(u) \\
\quad \times \exp \left[-\alpha^{2}\left(3 s^{2}+4 t^{2}+3 u^{2}\right) / 4\right] \\
\quad \times \sum_{n=0}^{\infty}(-1)^{n}(2 n+1) i_{n}\left(\alpha^{2} s t\right) i_{n}\left(\alpha^{2} t u\right) i_{n}\left(\alpha^{2} s u / 2\right) .
\end{aligned}
$$

Finally, the last diagram of interest corresponds to diagram 9 of figure 2. The evaluation procedure is quite similar to the previous case, and we merely write down its final expression

$$
\begin{aligned}
& D_{9}=(4 \pi)^{3} I(2 \alpha) \int_{0}^{\infty} s^{2} \mathrm{~d} s \int_{0}^{\infty} t^{2} \mathrm{~d} t \int_{0}^{\infty} u^{2} \mathrm{~d} u g(s) h(t) w(u) \\
& \times \exp \left[-3 \alpha^{2}\left(s^{2}+t^{2}+u^{2}\right) / 4\right] \\
& \times \sum_{n=0}^{\infty}(2 n+1) i_{n}\left(\alpha^{2} s t / 2\right) i_{n}\left(\alpha^{2} t u / 2\right) i_{n}\left(\alpha^{2} s u / 2\right) .
\end{aligned}
$$

\section{References}

[1] Bishop R F, Flynn M F, Boscá M C, Buendía E and Guardiola R 1990 Phys. Rev. C 421341

[2] Bishop R F, Flynn M F, Boscá M C, Buendía E and Guardiola R 1990 The Nuclear Equation of State part A, ed W Greiner and H Stöcker (New York: Plenum) p 605

[3] Bishop R F, Flynn M F, Boscá M C, Buendia E and Guardiola R 1990 Condensed Matter Theories vol 5, ed V C Aguilera-Navarro (New York: Plenum) p 253

[4] Kümmel H, Lührmann K H and Zabolitzky J G 1978 Phys. Rep. C 361

[5] Bishop R F and Kümmel H 1987 Physics Today 4052

[6] Bishop R F 1991 Theor. Chin. Acta 8095

[7] Bishop R F, Flynn M F, Buendía E, Boscá M C and Guardiola R 1990 J. Phys. G: Nucl Pan. Phys. 16 L61

[8] Ceuleneer R, Vandepeutte P and Semay C 1987 Phys. Lett. 196B 303

[9] Ceuleneer R and Vandepeutte P 1985 Phys. Rev. C 311528

[10] Ceuleneer R, Semay C and Vandepeutte P 1990 J. Phys. G: Nucl. Part. Phys. 161295

[11] McGrory J B and Wildenthal B H 1975 Phys. Lett. 60B S

[12] Afnan I R and Tang Y C 1968 Phys. Rev. 1751337

[13] Malfiet $\mathrm{R}$ A and Tjon $\mathrm{J}$ A 1969 Nucl Phys. A 127161

[14] Bishop R F, Buendía E, Flynn M F and Guardiola R 1991 J. Phys. G: Nucl Part. Phys. 17857

[15] Bishop R F, Buendía E, Flynn M F and Guardiola R 1991 Condensed Matter Theories vol 6, ed $S$ Fantoni and $S$ Rosati (New York: Plenum) p 405

[16] Boys S F 1960 Proc. $R$ Soc. A 258402

[17] Singer K 1960 Proc. R. Soc. A 258412 
[18] Longstaff J V and Singer K 1960 Proc. $R$ Soc. A 258421

[19] Bracci L, Rosati S and Viviani M 1987 Perspectives on Theoretical Nuclear Physics ed L Bracci et al (Pisa: ETS Editrice) p 34

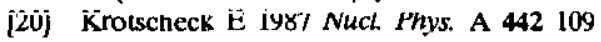

[21] Carlson J and Pandharipande V R 1981 Nuch. Phys. A 371301

[22] Tjon J A 1975 Phys. Leth 56B 217

[23] Fabre de la Ripelle M, Fiedeldey H and Sofianos S A 1988 Phys. Rev. C 38449

[24] Oehm W, Sofianos S A, Fiedeldey H and Fabre de la Ripelle M 1990 Phys. Rev. C 422322

[25] Oehm W, Sofianos S A, Fiedeldey H and Fabre de la Ripelle M 1991 Phys. Rev. C 4325

[26] Kievsky A, Viviani M and Rosati S 1990 Few-Body Syst. 91

[27] Kievsky A, Viviani M and Rosati S 1991 Condensed Matter Theories vol 6, ed S Fantoni and S Rosati (New York: Plenum) p 391

[28] Zabolitzky J G 1981 Phys. Lett. 100B 5

[29] Nakaichi-Maeda S, Akaishi A and Tanaka H 1980 Prog. Theor. Phys. 641315

[30] Akaishi Y, Chin S A, Horiuchi H and lkeda K 1986 lin. Rev. Nuch Phys. 4302

[31] Kameyama H, Kamimura M and Fukushima Y 1989 Phys. Rev. C 40974

[32] Zabolitzky J G and Kalos M H 1981 Nucl Phys. A 356114

[33] Killingbeck J P 1983 Microcomputer Quantum Mechanics (Bristol: Adam Hilger)

[34] Viviani M, Kievsky A and Rosati S 1991 Correlated hyperspherical harmonic calculations for threeand four-body systems Preprint University of Pisa

[35] Ballot J L 1981 Z. Phys. A 302347

[36] Bishop R F, Buendía E, Flynn M F and Guardiola R 1992 J. Phys. G: Nucl. Part. Phys. 18 L21

[37] Abramowitz M and Stegun I A 1968 Handbook of Mathenatical Functions (New York: Dover) 